\title{
Jeu de rôle sur table 2.0 : du salon aux plateformes de streaming
}

\section{Ugo Roux et Noémie Roques}

\section{OpenEdition}

1 Journals

Édition électronique

URL : http://journals.openedition.org/sdj/1449

DOI : $10.4000 /$ sdj. 1449

ISSN : 2269-2657

\section{Éditeur}

Laboratoire EXPERICE - Centre de Recherche Interuniversitaire Expérience Ressources Culturelles Education

\section{Référence électronique}

Ugo Roux et Noémie Roques, "Jeu de rôle sur table 2.0 : du salon aux plateformes de streaming », Sciences du jeu [En ligne], 10 | 2018, mis en ligne le 01 novembre 2018, consulté le 10 décembre 2020. URL : http://journals.openedition.org/sdj/1449; DOI : https://doi.org/10.4000/sdj.1449

Ce document a été généré automatiquement le 10 décembre 2020.

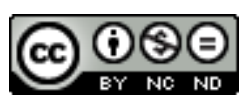

La revue Sciences du jeu est mise à disposition selon les termes de la Licence Creative Commons Attribution - Pas d'Utilisation Commerciale - Pas de Modification 4.0 International. 


\title{
Jeu de rôle sur table 2.0 : du salon aux plateformes de streaming
}

\author{
Ugo Roux et Noémie Roques
}

1 Le jeu de rôle sur table (aussi désigné indifféremment par le terme de jeu de rôle papier ou par le sigle JdR) est un jeu de société et de simulation qui propose aux rôlistes (pratiquants de jeu de rôle papier) d'y développer coopérativement une fiction ludique dans laquelle ils interprètent des personnages qui s'inscrivent dans le contexte du jeu à travers un large espace d'appropriation. Une partie de jeu de rôle papier nécessite un nombre minimum de deux rôlistes (dont un maître du jeu) pour créer le cadre du jeu par un échange verbalisé (le plus souvent oralisé) ; il s'agit de la condition sine qua non pour actualiser le contexte imaginaire (Shulga, 2003) et développer la fiction ludique à partir d'une situation initiale. Le maître du jeu assure la médiation entre les joueurs et l'univers de jeu; il a traditionnellement la gestion de tout l'univers de jeu extérieur aux personnages des joueurs et de sa cohérence. Grâce au personnage, réel dispositif et vecteur immersif (Caïra [2007] parle de « véhicule») dans l'univers fictionnel, le joueur peut faire évoluer la fiction ludique. C'est donc par le biais de l'action virtuelle (dont la résolution se fait généralement avec des dés) de leurs personnages que les joueurs interagissent avec l'univers de jeu selon leurs désirs dans les limites imposées par le besoin de cohérence (Fine, 2002).

2 Cet article s'intéresse à l'évolution de la pratique du jeu de rôle papier à l'ère du numérique et donc à l'imprégnation de cette dernière sur ce jeu. Comme pour de nombreuses autres activités et pratiques, les technologies de l'information et de la communication semblent s'immiscer parfois dans la pratique du jeu de rôle papier, ce qui a pour effet de faire naitre de nouveaux usages et comportements et de modifier les logiques de ce jeu (Roux, 2016). Parmi ces nouveaux usages et comportements, il est notamment observable depuis quelques années des diffusions en streaming ${ }^{1}$ de parties de jeu de rôle sur table. ${ }^{2}$ Nos recherches font remonter la première diffusion de ce genre d'émissions filmées au 5 septembre 2010 avec le programme PAX Prime 2010 D\&D Game d' Acquisitions Incorporated (sources: https://youtu.be/uqXqK3ZlqWI et http://www.acqinc.com/portfolio). 
Cesar et Geerts (2011) désignent ces nouveaux dispositifs de médiatisation par le terme de « télévision sociale ». Parmi eux, « le streaming est un contexte extrêmement intéressant pour les médias en ligne participatifs [...] qui a mis le consommateur traditionnel dans le rôle de créateur de contenus " (Sjöblom et Hamari, 2016). Le streaming, tel que nous l'entendons en tant qu'activité culturelle, se distingue des autres formes de diffusion de contenus vidéo en ligne par son haut niveau d'interaction qui en fait un canal communicationnel plus complet (Sjöblom et Hamari, 2016). Ce dispositif permet notamment aux viewers (terme généralement employé pour désigner les spectateurs de streamings) d'interagir entre eux mais aussi parfois avec les créateurs de contenus, et ce, souvent en temps réel (cas du live streaming). Le streaming étend ainsi à une audience les cadres de participation ainsi que les plans de présence du jeu de rôle papier (voir Fine [2002] et Shulga [2003] pour ces deux notions).

Ce constat nous amène à nous demander comment cette pratique du streaming redéfinit l'espace de jeu dont les frontières peuvent parfois devenir ténues et poreuses avec le hors-jeu en intégrant le spectateur dans l'action ludique de manière toujours plus interactive. Cette question nous conduit par conséquent à nous demander comment le spectateur s'inscrit dans cette nouvelle logique ainsi qu'à mobiliser la notion de « spectacteur» (Dumouchel, 1991).

5 Pour répondre à ces questions, nous proposons d'observer les nouveaux comportements et usages ainsi que les nouvelles logiques issus de la médiatisation du jeu de rôle sur table par le streaming. Pour mener à bien cette observation et récolter les données nécessaires à cet objectif, nous avons soumis un questionnaire en ligne (du 4 au 25 mars 2018) à de nombreux sujets (580 participants) principalement de nationalité française et canadienne (québécoise). Notre enquête a été diffusée sur des médias socionumériques dédiés au jeu de rôle sur table (e.g. des groupes Facebook comme Discussions de rôlistes, Québec jeux de rôle sur table, RDF Rôliste de France ou encore Union Rôliste Francophone, des forums comme Opale Rôliste ou celui de la Fédération Française de Jeu De Rôle). Il s'agissait d'un questionnaire réalisé sur Google Forms avec 40 items qui interrogeait les participants essentiellement sur leurs habitudes de consommation d'émissions de parties de jeu de rôle sur table. L'étude portait également sur les éventuelles conséquences de cette consommation, qu'elles soient sociales ou ludiques. Les participants au questionnaire étaient joueurs de jeu de rôle sur table (qu'ils soient spectateurs ou non d'émissions en streaming de parties de jeu de rôle papier), usagers des médias socionumériques et inscrits sur certains d'entre eux. Leurs réponses dessinent des tendances que nous présenterons, mais dont le caractère généralisable à l'ensemble de la population rôliste consommatrice d'émissions de parties de jeu de rôle sur table reste à vérifier dans la mesure où celles-ci portent sur l'échantillon particulier qui était le nôtre, à savoir des rôlistes connectés et inscrits sur des médias socionumériques. Les conclusions qui se dégagent de ces tendances particulières ne sont donc pas extrapolables à l'ensemble de la population rôliste, en particulier celle qui n'est pas « connectée ».

\section{La « spectatorisation » du jeu de rôle sur table}

Le jeu de rôle est peu connu cependant. Il est tourné vers l'intérieur, pratiqué par de petits groupes dans l'intimité du domicile privé. Il échappe à la connaissance informelle que tout acteur social peut avoir des activités des «autres " (qu'est-ce que les gens font?), faite d'informations directes et personnelles, d'expériences communes, de simples regards et de médiatisation. Cette faible visibilité tient sans 
doute aussi à son mode de diffusion: loin du prosélytisme, il s'insinue par « initiation »- mot consacré des joueurs pour désigner leur premier apprentissage du jeu - et sans être secrète, c'est une pratique peu bavarde sur elle-même, dont les joueurs ne font pas ostentation. (Voelckel, 1995, p. 58).

6 Le jeu de rôle sur table est un "petit monde social» (Fine, 2002) avec une présence médiatique qui se structurait jusqu'à récemment principalement autour d'une presse, de podcasts, de forums et autres sites Internet tous spécialisés, mais qui demeurait faiblement tournée vers l'extérieur à l'image de sa pratique (Beau, 2007). Or, cet « entresoi rôliste" semble s'assouplir. En effet, une activité connexe à celle des streameurs (individus qui font du streaming) de jeux vidéo se développe. Il s'agit de la diffusion - en direct (live streaming en anglais) ou enregistrée - de parties de jeu de rôle papier sur des plateformes telles que Twitch ou YouTube (e.g. Donjons \& Jambons, Aventures, Les Chroniques du Necronomicon en France, Critical Role aux États-Unis, Dans le Donjon d'Es-Tu Game au Québec/Canada, Yogsquest au Royaume-Uni). Le jeu se rapproche, dans ce cas bien précis, de la notion de semi-loisir ${ }^{3}$ développée par Dumazedier (1974) puisqu'il répond alors à d'éventuels impératifs d'audience et de notoriété. Par conséquent, le maître du jeu adopte fréquemment une posture de maître de cérémonie puisque son public n'est plus seulement constitué de ses joueurs.

7 Loin de se contenter de seulement filmer le jeu et son cadre, ces émissions se distinguent parfois par une sophistication poussée de ce que Caïra (2007) appelle le "montage ", à savoir le degré de préparation d'une partie de jeu de rôle sur table. Alors que ce degré est très variable, celui-ci peut parfois se rapprocher d'un phénomène de spectacularisation pour certaines émissions. À l'instar du sport électronique (e-sport en anglais), le jeu est rendu plus attractif, tant pour les joueurs que pour les spectateurs (Besombes, 2015). En effet, entre génériques et réalisation soignés, costumes travaillés, angles de caméras optimisés et autres moyens mobilisés (e.g. studios d'enregistrement pleinement équipés, équipes techniques), ces émissions adoptent les codes de certains formats télévisuels.

Ces émissions intègrent volontairement dans le jeu le spectateur comme une troisième partie prenante dont il faut créer et maintenir l'engagement d'une nouvelle manière puisqu'elle n'est pas actrice dans la diégèse, d'autant plus qu'elle est plongée dans la même "pauvreté sensorielle» que les joueurs et le maître du jeu (Caïra, 2007). L'intégration du spectateur devient dès lors un des enjeux et l'une des exigences de divertissement du "contrat» qui lie les rôlistes sur la bonne manière de conduire la partie (Caïra, 2007). Par exemple, alors que les moments parasites (e.g. les dialogues « méta- ») sont fréquents dans une partie de jeu de rôle papier, ceux-ci peuvent nuire à la partie par leur excès (Fine, 2002 ; Caïra, 2007). Pour y remédier les rôlistes peuvent tenter d'éviter les moments de forte digression qui pourraient se révéler perturbateurs pour le spectateur. Dans certaines émissions enregistrées ces moments sont délibérément retirés au montage pour éviter un ralentissement du rythme qui pourrait faire chuter l'attention et l'intérêt du spectateur.

9 Ainsi, la pratique du jeu de rôle sur table, qui se voulait intimiste, investit - à grand renfort de moyens techniques et esthétiques - les médias socionumériques et s'ouvre à une audience distante dans l'espace et parfois le temps. 


\section{Les médias socionumériques comme porte d'entrée dans le jeu}

10 Notre enquête révèle qu'une large majorité des répondants $(87,8 \%, \mathrm{~S}=509)$ connaissait déjà l'existence d'au moins une émission en ligne (en streaming) qui met en scène une partie de jeu de rôle sur table avant de participer à ce questionnaire (question: "Connaissiez-vous l'existence des émissions en ligne (en streaming) présentant une partie de jeu de rôle sur table avant de participer à ce questionnaire? " [S = 580]). D'ailleurs, nombreux sont les répondants à avoir découvert l'existence de ces émissions grâce aux médias socionumériques et aux technologies de l'information et de la communication. Le premier vecteur de promotion de celles-ci apparaît être les réseaux sociaux numériques (figure 1 ; question: "Comment avez-vous connu ces émissions?" [S=509]). Parmi ceux qui connaissaient auparavant leur existence, la part de ceux qui ont déjà regardé ce genre d'émissions s'élève à $86,2 \%(\mathrm{~S}=439)$ (question: "Avez-vous déjà regardé une de ces émissions?" [S=509]). Dans le panorama des émissions les plus connues, les plus regardées et qui ont le plus d'abonnés nous pouvons notamment citer Aventures (émission française par Bazar du Grenier), Donjons \& Jambons (émission française par O'Gaming), Critical Role (émission américaine par Geek \& Sundry), Les Chroniques du Necronomicon (émission française par Globtopus), Les Aventureux (émission canadienne/québécoise par Les Aventureux), entre autres (figure 2; questions : "Quelle(s) émission(s) connaissez-vous?» $[S=509]$; «Quelle(s) émission(s) avez-vous déjà regardée(s) ? » $[S=439]$; «À quelle(s) émission(s) êtes-vous abonné(e) ?" [S=439]).

Figure 1

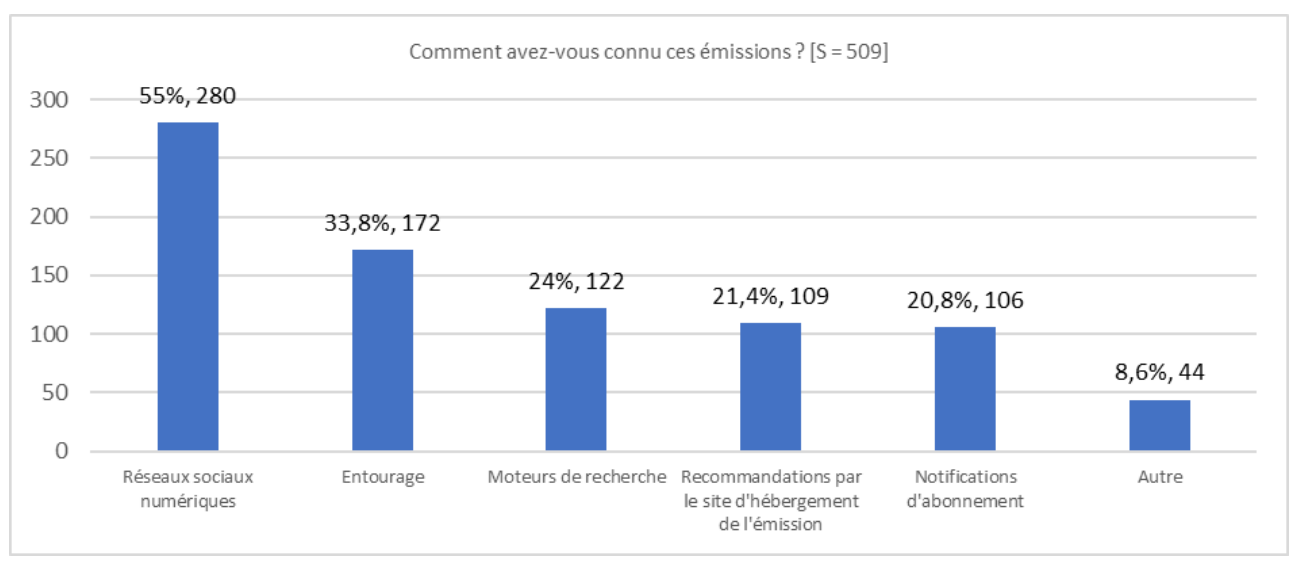

Les moyens de découverte des émissions de parties de jeu de rôle sur table 
Figure 2

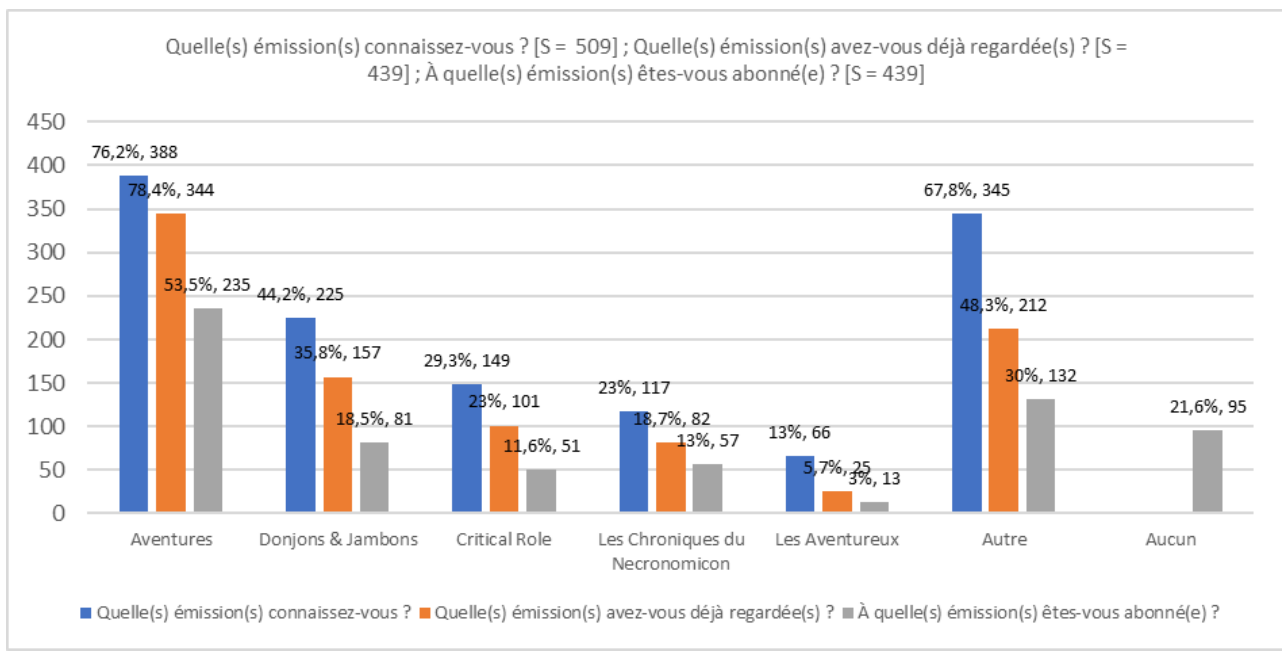

Les émissions de parties de jeu de rôle sur table les plus connues

11 Les espaces conversationnels mis à disposition en parallèle de ces émissions se révèlent parfois être des lieux de socialisation. Des répondants $(18,2 \%, \mathrm{~S}=80)$ déclarent avoir développé leur environnement social après participation « spectactorielle » à certaines de ces émissions (question: "Cette participation a-t-elle contribué au développement de votre environnement social (intégration d'un groupe ou d'une communauté rôliste, liens d'amitié avec d'autres rôlistes, etc.)?» $[\mathrm{S}=439])$. Nous pouvons distinguer trois grandes méthodes de développement de l'environnement social rôlistique de l'individu.

La première de ces méthodes est la recherche de joueurs déjà "initiés " (comme par exemple via des annuaires spécialisés). Les médias socionumériques apparaissent aujourd'hui être l'un des moyens privilégiés pour développer l'environnement social rôlistique de l'individu ; par exemple la figure 3 présente un billet datant du 25 mars 2018 posté dans le groupe Facebook «Discussions de Rôlistes" où l'auteur déclare chercher des partenaires de jeu (source : https://www.facebook.com/groups/608825622473156/).

Figure 3

Hey oh!

Je suis un joueur plutôt sympa qui cherche une table sur Bordeaux afin de découvrir de nouveaux horizon (nouveaux comme je n'ai pas pu tenter grand chose)

Ou alors des parties sur Roll20. Bref, je suis quelqu'un qui cherche à continuer sa passion!
(3) J'aime
Commenter
Partager

Publication dans le groupe Facebook « Discussions de Rôlistes » où l'auteur déclare chercher des partenaires de jeu. Source : https://www.facebook.com/groups/608825622473156/ 
13 Concernant cette méthode, les émissions se révèlent être un moyen efficace de promotion du jeu de rôle sur table auprès des " profanes ». En effet, celles-ci permettent d'éviter de longues et fastidieuses explications descriptives de ce qu'est le jeu de rôle papier pour se concentrer sur le jeu en lui-même (son univers, sa mécanique, son ambiance, etc.) tout en proposant un format de découverte moins engageant qu'une éventuelle première partie.

La troisième méthode est le prétexte à discussion. Cette méthode peut se suffire à ellemême ou bien se fondre dans les deux précédentes. Les émissions représentent un excellent prétexte à discussion autour du jeu, que ce soit entre amis ou avec des inconnus, en club ou dans les médias socionumériques, etc. Les plateformes où sont hébergées ces émissions proposent généralement des espaces publics de prise de parole pour satisfaire ce prétexte à discussion (par exemple la figure 4 présente une conversation tenue dans la partie réservée aux commentaires d'un épisode de la série Aventures: AVENTURES \#1 L'inconnue [source : Bazar du Grenier; https://youtu.be/ZJ_uZSurIBM]). De ces discussions peuvent fréquemment découler l'intégration d'un nouveau groupe de joueurs, le tissage de nouveaux liens d'amitié, etc.

Figure 4

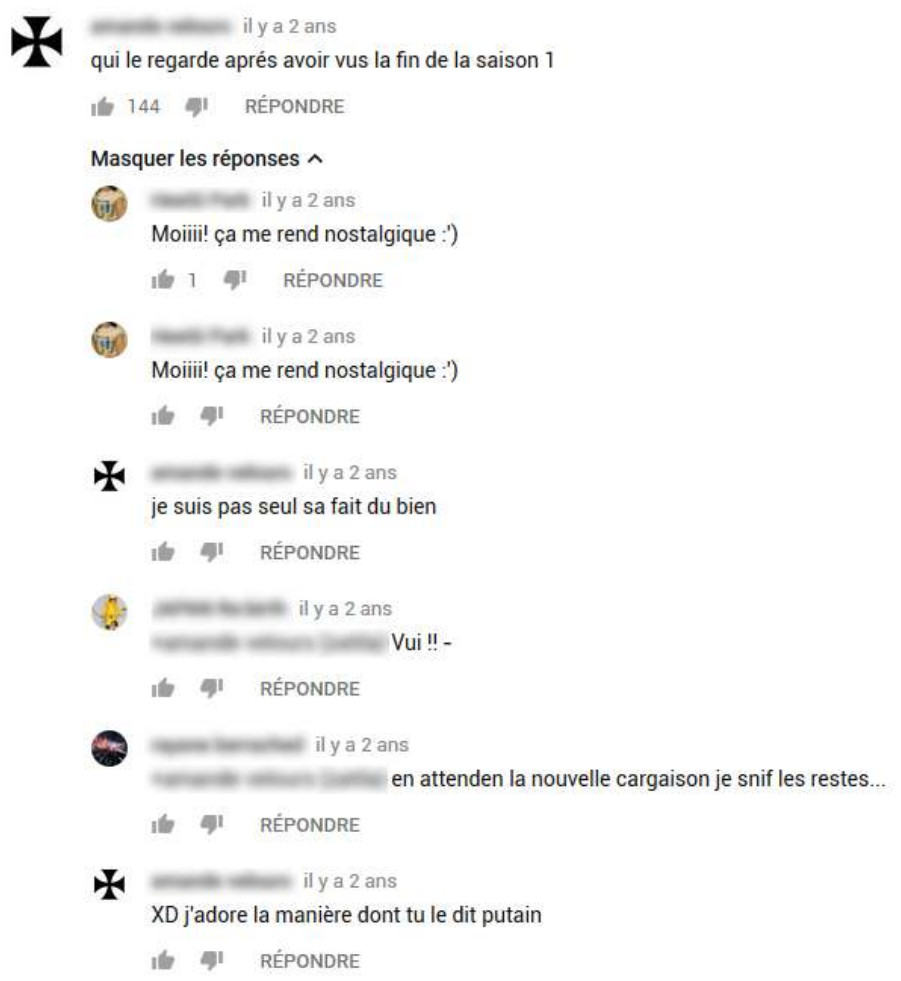

Conversation tenue dans la partie réservée aux commentaires d'un épisode de la série Aventures : AVENTURES \# 1 - L'inconnue. Source : Bazar du Grenier; $h$ ttps://youtu.be/ZJ_uZSurIBM

Il est également intéressant d'observer que certains des spectateurs $(8,9 \%, \mathrm{~S}=39)$ ont découvert le jeu de rôle papier grâce à ces dernières (question : "Ces émissions vous ontelles fait découvrir le jeu de rôle sur table?» [S = 439]). La série Aventures de Bazar du Grenier (chaîne secondaire de Frédéric Molas et Sébastien Rassiat) est d'ailleurs l'émission la plus citée par nos répondants pour avoir été celle à l'origine de cette découverte (figure 5 ; question: "Quelle émission vous a fait découvrir le jeu de rôle sur table?» $[\mathrm{S}=39])$. Nous 
pouvons raisonnablement penser que ce résultat s'explique par le fait que l'émission Aventures $^{4}$ tire grandement profit de la chaîne YouTube de Joueur du Grenier (chaîne principale de Frédéric Molas et Sébastien Rassiat), de son succès, de sa notoriété, de sa popularité et de sa fan base considérable (Joueur du Grenier cumule plus de 3 millions d'abonnés et plus de 560 millions de vues tandis que Bazar du Grenier cumule plus de 1,2 million d'abonnés et plus de 350 millions de vues). Bazar du Grenier - et par extension Joueur du Grenier - se pose en média majeur du jeu de rôle sur table dans la sphère des médias socionumériques francophones grâce à Aventures.

Figure 5

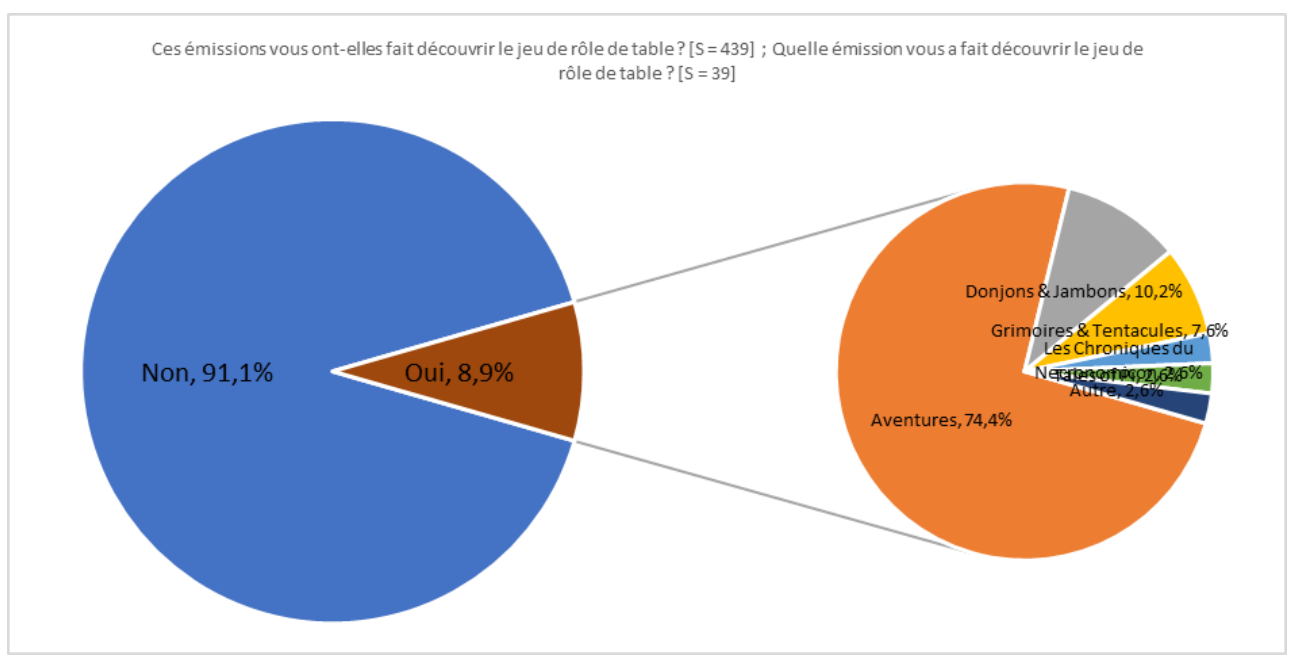

Les émissions qui ont principalement fait découvrir le jeu de rôle sur table

Pour une grande majorité de ceux qui ont découvert le jeu de rôle sur table avec ces émissions $(87,2 \%, S=34)$, cette découverte sous ce format a eu pour effet d'en faire des "primo-joueurs", c'est-à-dire que ces émissions ont suscité chez eux un intérêt tel qu'elles les ont poussés à jouer pour la première fois au jeu de rôle sur table (question: "Ces émissions vous ont-elles poussé à jouer pour la première fois au jeu de rôle sur table?" $[S=39]$ ). Ces émissions peuvent dans ce cas se situer en amont de la première étape de ce que Trémel (2001) appelle la " carrière du joueur » et qui consiste en l'intégration d'une communauté de joueurs. Elle la précède dans la mesure où elle ne constitue pas en tant que telle l'intégration de cette communauté de joueurs par l'individu mais plutôt un éventuel premier point de contact avec cette dernière. D'ailleurs, pour trouver leur première partie de jeu, les primo-joueurs se sont principalement tournés vers leur entourage (figure 6 ; questions : "Si oui, où avez-vous cherché votre première partie de jeu de rôle sur table?" [S=34]; "Où avez-vous finalement trouvé votre première partie de jeu de rôle sur table?» $[\mathrm{S}=34])$. 
Figure 6

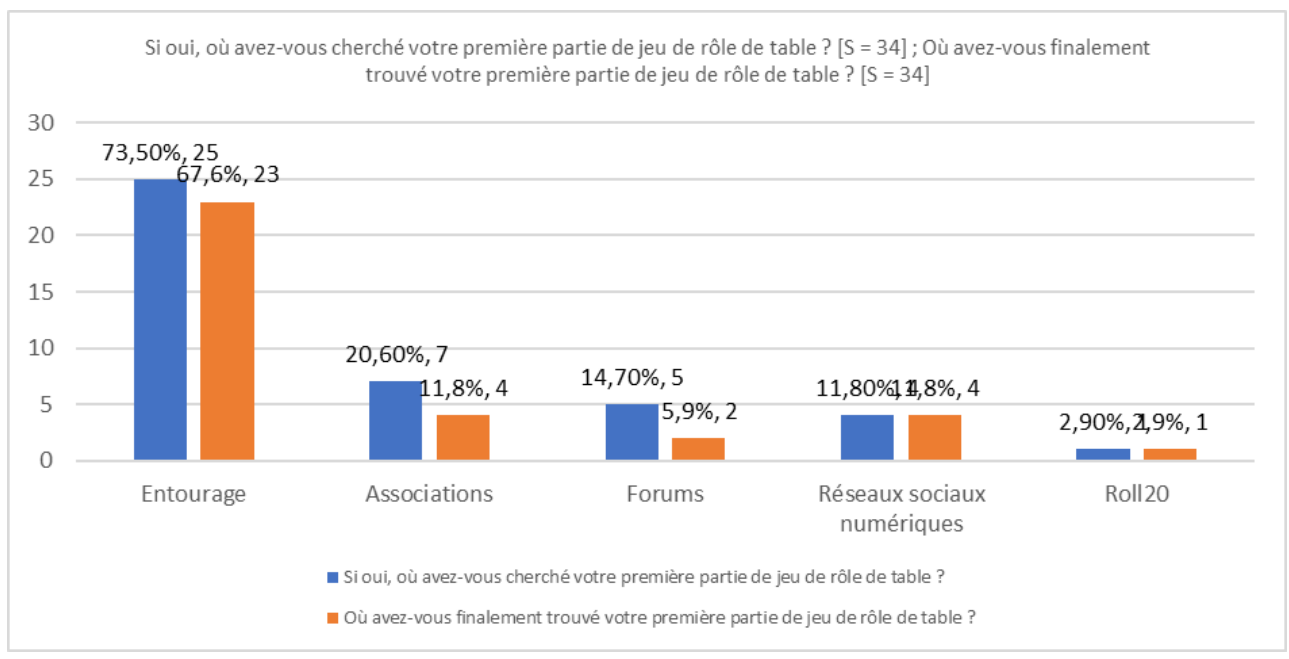

Les moyens de recherche d'une première partie de jeu de rôle sur table pour les « primo-joueurs »

Enfin, ces émissions ont modifié favorablement l'opinion que pouvaient avoir ceux qui parmi nos répondants ne connaissaient pas auparavant le jeu de rôle papier. En effet, parmi ceux qui ne connaissaient pas le jeu de rôle sur table avant de le découvrir dans une de ces émissions, $56,4 \%(\mathrm{~S}=22)$ en avaient une opinion favorable, $5,1 \%(\mathrm{~S}=2)$ en avaient une opinion défavorable, $23,1 \%(\mathrm{~S}=9)$ en avaient une opinion neutre et $15,4 \%$ $(\mathrm{S}=6)$ n'avaient pas d'opinion à son sujet (question : "Quelle était votre opinion sur le jeu de rôle sur table avant de le découvrir dans ce type d'émissions? " [S = 39]). Tous les précédents répondants déclarent avoir désormais une opinion favorable à propos du jeu de rôle sur table. Il faut cependant écarter toute conclusion hâtive, ce résultat étant attendu puisqu'il porte sur des individus qui jouent désormais au jeu de rôle papier et qui fréquentent des médias socionumériques dédiés à ce jeu. On ne peut donc tenter d'extrapolation de ces résultats à l'ensemble des spectateurs d'émissions en ligne de parties de jeu de rôle sur table, en particulier ceux qui ont gardé une opinion défavorable de ce jeu.

\section{Vers une miscibilité des cadres de participation?}

Comme le soulignait Granjon (2012, p. 126), «la médiation de l'informatique connectée déspatialise et désynchronise l'acte de monstration et le moment de sa réception ». La plateforme d'hébergement de vidéos YouTube a fait, comme tout média socionumérique, de la culture participative l'un de ses principaux moteurs (Burgess et Green, 2009). À ce titre, YouTube accorde notamment à ses usagers la possibilité de réagir aux vidéos qu'ils visionnent à travers des commentaires qu'il leur est possible de laisser dans un espace dédié. Pour leur part, les Youtubeurs (individus créateurs de contenus vidéo sur YouTube) multiplient les occasions et les dispositifs pour échanger avec leur audience lorsqu'ils sont en différé (pour, par exemple, apporter des explications sur des problèmes techniques rencontrés, clarifier des situations ou encore résumer la progression de précédentes séances de jeu). Ils partagent ainsi avec leur public ce que Beaudouin (2002) appelle une " coprésence écranique ». Cette forme de coprésence consiste à " partager un même contexte, une même situation définie par un ensemble de textes disponibles à l'écran» (Beaudouin, 2002, p. 219). Le live streaming tend à réduire la désynchronisation 
communicationnelle générée par les distances spatiales et temporelles induites par le streaming « ordinaire ».

Plusieurs cadres de participation s'emboîtent dans le jeu de rôle sur table (Fine, 2002 ; Shulga, 2003). Ces cadres vont de l'espace commun de communication au contexte imaginaire de jeu où la fiction prend vie. Ces cadres produisent « un espace combiné de présence dans lequel l'interaction des joueurs se déroule et à l'intérieur duquel ils passent constamment d'un plan de présence à un autre» (Shulga, 2003, p. 106). Ces plans de présence sont au nombre de trois :

L'action de jeu qui représente le plan le plus immersif où se déroulent les événements intradiégétiques et où le roleplay peut se développer; l'action ludique qui représente le plan métafictionnel où la fiction est commentée de façon extradiégétique mais toujours dans un but ludique; le cadre réel où se produisent des événements extérieurs au jeu et donc sans but ludique. (Roux, 2016).

Roux (2016) constatait que les technologies de l'information et de la communication modifient la logique des cadres de participation du jeu de rôle papier et les redéfinissent dans leur spatialisation et leur temporalité. Un de ces cadres de participation en particulier se voit parfois totalement redéfini : l'espace commun de communication. Ce cadre de participation se caractérise à l'origine par la coprésence physique des joueurs dans un même espace réel afin d'assurer une communication synchrone et présentielle (Roux, 2016). De plus, Sjöblom et Hamari (2016) remarquaient que la consommation de streamings de jeux vidéo est une forme non-intuitive de consommation de médias pour le spectateur et nous pouvons raisonnablement penser qu'il en va de même pour le streaming de jeux de rôle sur table. En effet, les jeux sont une forme de médias hautement interactive et la satisfaction engendrée par ce média repose sur l'interaction directe et active avec le jeu (Sjöblom et Hamari, 2016). Dans le cas du streaming de jeux de rôle papier, cette interaction semble à première vue absente entre le spectateur et le jeu. Or, au-delà d'étendre l'espace commun de communication à l'intégration de spectateurs en distanciel, l'intention des streameurs est de faire participer ces mêmes spectateurs au jeu en les intégrant dans l'action ludique et en leur permettant, par exemple, d'être décisionnaires sur certaines tournures des événements du scénario (dans le cas particulier du live streaming). Sans être joueur le spectateur devient tout de même un des participants au jeu; sa posture est plus passive que celle du joueur mais elle est toutefois plus active que celle du simple spectateur (Sjöblom et Hamari, 2016).

\section{Du spectateur au « spectacteur » ou l'intégration du hors-jeu dans le jeu}

21 Les frontières spatiales et temporelles du jeu de rôle sur table sont fréquemment redessinées avec les technologies de l'information et de la communication. Le streaming, et plus particulièrement le live streaming, contribue à cette redéfinition d'une façon singulière puisqu'il a non seulement ouvert l'espace commun de communication et d'interaction à des spectateurs distants géographiquement, et il a également étendu à ceux-ci deux des trois plans de présence définis par Fine (2002) et Shulga (2003) : l'action ludique et le cadre réel. Cette extension à ces deux plans de présence, et plus particulièrement à l'action ludique, font passer les spectateurs d'une posture passive à une posture active et participative. Ils sont transformés ainsi en « spectacteur ».

Dumouchel dit de la notion de spectacteur qu'elle 
est un mot-valise contractant le mot spectateur et le mot acteur. Le spectacteur est un spectateur-acteur de l'œuvre interactive. Il est à la fois l'observateur du récit, celui pour qui le récit est dit ou montré, et le protagoniste du récit, celui par qui le récit se joue. (Dumouchel, 1991, p. 41).

Cette définition place le spectacteur au cœur de l'œuvre interactive puisqu'il en serait le protagoniste. Pourtant l'auteur reconnaît qu'il s'agit là d'une vision idéalisée de cette posture puisque «quand une œuvre n'a d'interactive que le nombre limité d'options qu'elle laisse au public, nous nous trouvons bien près du degré minimum d'interactivité. » (p. 41). Il existe donc un « gradient » d'interactivité spectactorielle.

Dans le cas du jeu de rôle sur table, on peut dégager différents protagonistes selon les plans de présence où l'on se positionne. Dans la diégèse - plan auquel n'ont pas accès les spectacteurs de façon interactive puisqu'ils n'ont pas de personnages - il s'agit sans aucun doute des personnages des joueurs tandis que dans l'action ludique ce sont les joueurs et le maître du jeu qui endossent conjointement ce rôle. L'opportunité est parfois offerte aux spectacteurs de se positionner en tant que protagonistes en se substituant au maître du jeu en lui donnant accès de façon interactive au plan de présence de l'action ludique. Le cadre réel quant à lui ne dispose pas à proprement parler de protagonistes.

Weissberg (1999) se saisit de la notion de spectacteur à l'heure des dispositifs «transmédias »..$^{5}$ Il dit de ceux-ci qu'ils inversent les positions des spectateurs et les transforment en spectacteurs. L'emphase est alors mise sur les possibilités d'interaction de l'individu plutôt que sur sa position de protagoniste. Rebillard (2007) et Schmitt (2015) s'interrogent sur la nature des interactions spectactorielles possibles à travers ces nouveaux dispositifs. Sont-elles de l'ordre de la contribution, de la création ou du commentaire? Qu'en est-il dans le cas des émissions en streaming présentant des parties de jeu de rôle papier ? Pour le vérifier nous avons posé aux participants de notre enquête la question suivante : "En tant que spectateur, avez-vous déjà participé à une de ces émissions selon les modalités qui suivent?" $(\mathrm{S}=439)$. À cette question nous leur avons proposé un certain nombre de modalités de réponse (figure 7). Il semblerait finalement que la participation des spectacteurs de streaming de jeux de rôle sur table soit principalement de l'ordre des trois catégories d'activités précédemment citées.

Figure 7

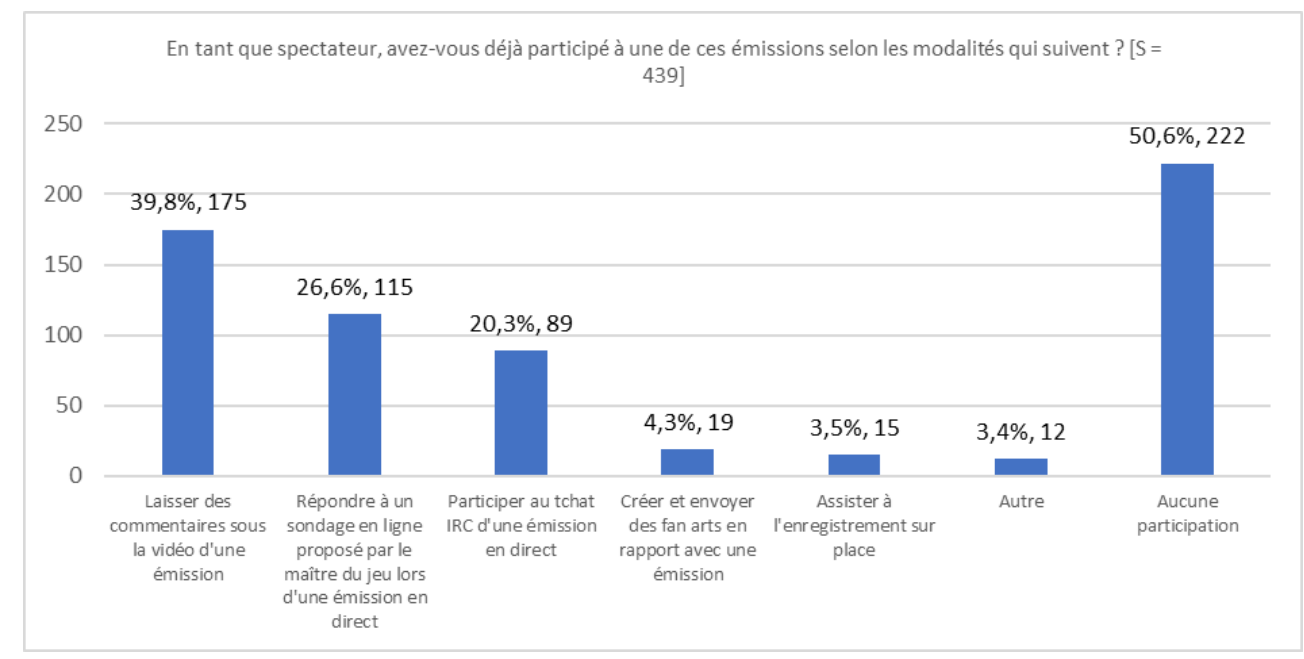

Volume et nature des participations "spectactorielles" 
enquête montre que l'activité spectactorielle la plus répandue dans le streaming de jeux de rôle papier est le commentaire (figure 7). Les manifestations les plus remarquables de cette activité se produisent à travers les commentaires laissés de façon quasi-synchrone $^{6}$ dans les tchats IRC (fenêtres de clavardage) des live streamings ou ceux laissés de façon asynchrone dans la partie commentaires des plateformes d'hébergement de vidéos (comme par exemple sous chaque vidéo publiée sur YouTube). 39,8 \% ( $\mathrm{S}=175)$ des répondants affirment avoir déjà laissé des commentaires sous la vidéo d'une émission et $20,3 \%(S=89)$ déclarent avoir déjà participé au tchat IRC d'une émission en direct. Ces commentaires se situent à un niveau extradiégétique et métafictionnel (sans que ceux-ci aient une quelconque incidence sur l'action ludique), voire fréquemment à un niveau extraludique qui se situe dans le cadre réel (voir la figure 8 qui présente la lecture littérale et immédiate, par les joueurs en fin de chaque épisode, des commentaires laissés par les viewers présents sur le tchat IRC de l'émission live de Donjons \& Jambons).

Figure 8

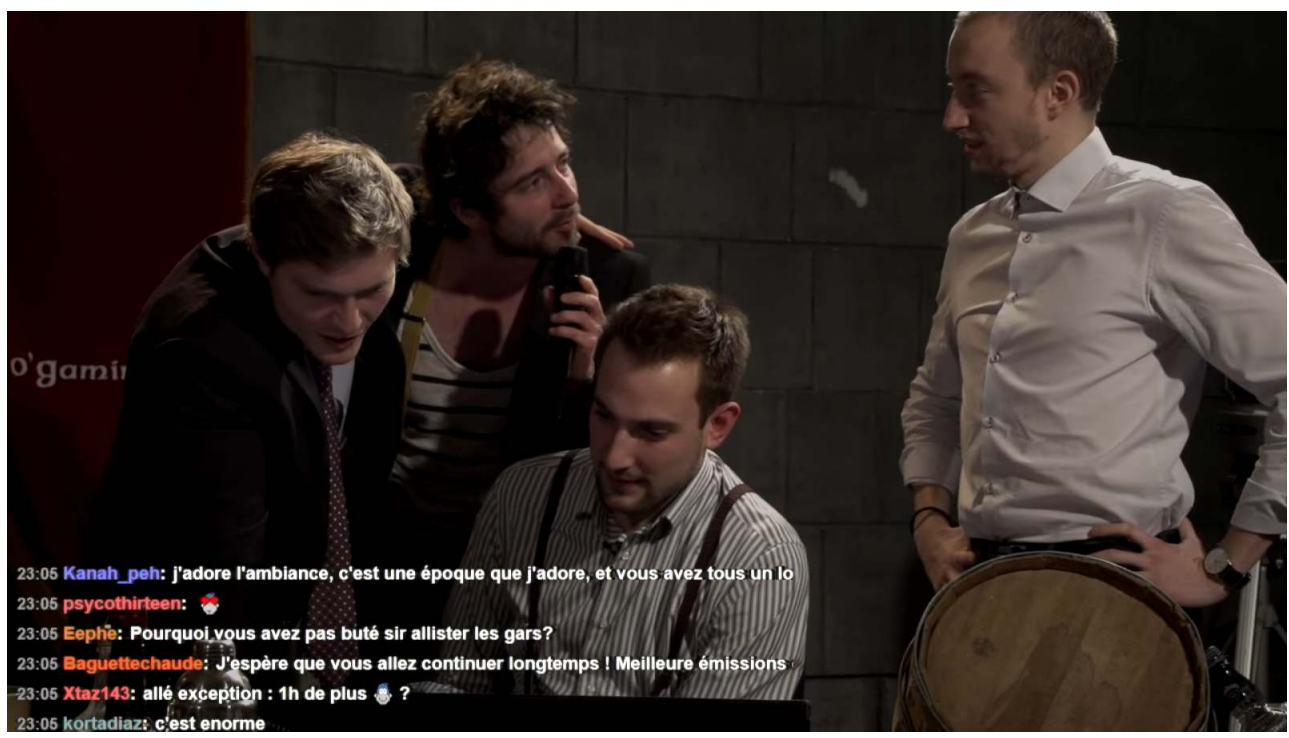

Commentaires laissés par les viewers présents sur le tchat IRC de l'émission live de Donjons \& Jambons : Les années folles - Épisode deuxième. Source : O'gaming TV; https://youtu.be/K1Arki82nHQ

La deuxième activité spectactorielle la plus notable dans le streaming de jeux de rôle papier semble être la contribution (figure 7). Il est fréquent dans les live streamings de jeux de rôle sur table que les streameurs fassent appel en cours de partie à leurs viewers pour orienter le scénario. Le maître du jeu redistribue un peu de son autorité au spectacteur afin de renforcer son engagement. Le maître du jeu étend ainsi son rôle central de médiateur à la médiation joueurs/spectacteurs. Le moyen le plus fréquemment utilisé pour cela est de recourir à un sondage en temps réel qui propose diverses possibilités d'issues à une situation donnée. La figure 9 présente les résultats d'un Straw Poll lancé durant un live de la série Aventures; la proposition remportant le vote est alors appliquée en jeu dans les minutes qui suivent la fin du suffrage. $26,6 \%(S=115)$ des participants répondent avoir déjà participé au tchat IRC d'une émission en direct. À l'instar des commentaires, cette activité de contribution se situe à un niveau extradiégétique et métafictionnel. Cependant, à l'inverse des commentaires, la contribution a un impact direct sur l'action ludique puisqu'elle influence le déroulement du scénario et donc la narration. Nous pouvons également citer pour exemple l'émission québécoise/ 
canadienne Dans le Donjon d'Es-tu Game qui se déroule en coprésence physique de ses spectateurs. Ses participants directs (maître du jeu et joueurs) demandent à leurs spectateurs d'écrire sur trois papiers les noms d'une "personnalité connue masculine », d'une "personnalité connue féminine " et d'un "personnage de fiction "; lorsqu'un personnage non-joueur est introduit, le maître du jeu pioche alors un papier et interprète le personnage selon le nom qui y est inscrit.

Figure 9

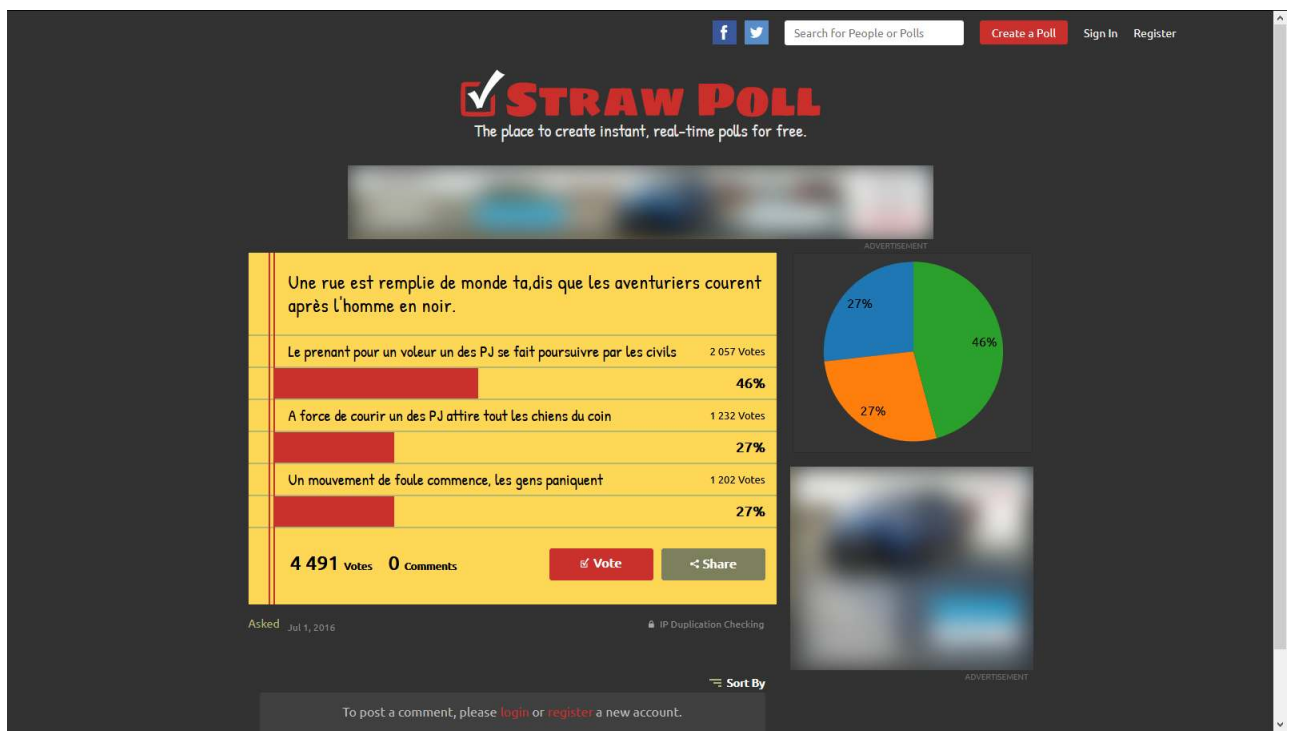

Straw Poll de l'émission Aventures Live : « Un nouvel aventurier » - (01/07/2016) - Partie 07 (Source Straw Poll : http://www.strawpoll.me/10640598/r/ Source vidéo : Bazar du Grenier ; https://youtu.be/ j63Ega_dMaY)

Enfin, la dernière activité spectactorielle - la création - est certainement celle qui est la moins représentée. Il arrive parfois que les streameurs sollicitent leurs spectateurs afin que ces derniers participent au jeu à travers une activité créatrice. C'est notamment le cas de l'émission américaine Critical Role qui invite ses viewers à dessiner les personnages du jeu qu'elle retransmet (ce que l'on appelle communément du fan art) ; certains de ces dessins sont ensuite retenus pour apparaître dans l'émission (voir la figure 10 qui présente une galerie de personnages dessinés par des spectateurs pour l'émission Critical Role). 4,3\% ( $\mathrm{S}=19)$ des répondants disent avoir déjà créé et envoyé des fan arts d'une émission. Ces créations, lorsqu'elles sont exploitées, peuvent contribuer à l'ambiance en augmentant l'expérience ludique des joueurs et l'expérience spectatorielle des spectateurs. La création s'inscrit dans les différents cadres de participation et plans de présence en fonction de sa nature. Si l'on reprend l'exemple du fan art, celui-ci pourrait avoir un impact sur la fiction s'il influence la création d'un personnage. En revanche, il n'aura qu'un intérêt illustratif s'il s'inspire d'un personnage déjà créé. 
Figure 10

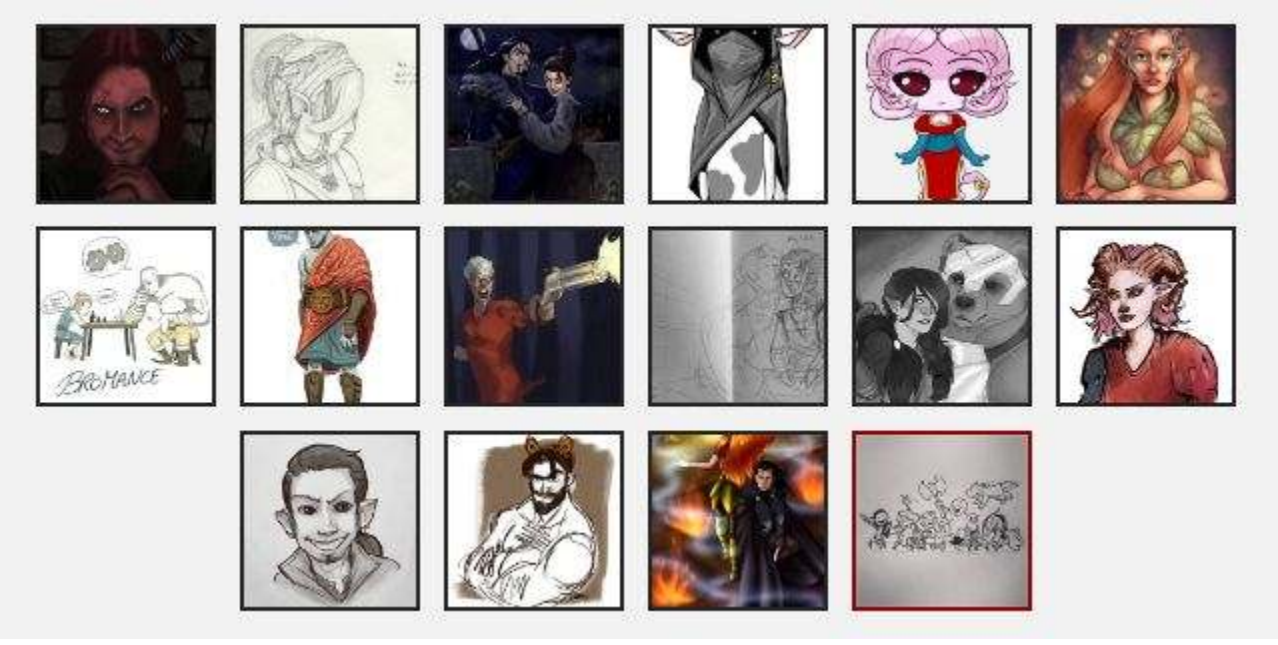

Galerie de fan arts pour l'émission Critical Role. Source: Geek \& Sundry - http://geekandsundry.com/ draw-for-initiative-critical-role-fan-art-gallery/?gallery=242316\#gallery

\section{Conclusion}

Dans le présent article nous avons pu observer comment le jeu de rôle sur table, via le streaming, étend ses plans de présence à une audience distante dans l'espace et parfois aussi dans le temps, et comment il étend ses cadres de participation à celle-ci également. Cela nous a conduit dans un premier temps à nous saisir de la notion de "spectacteur ». Cette extension s'accompagne en effet d'une volonté d'intégration interactive du spectateur parfois jusqu'à l'action ludique. Par cette intégration, le spectacteur prend parfois part à la « division des tâches » (Caïra, 2007) que suppose le travail collectif généré par le jeu de rôle sur table. Dans un second temps nous avons analysé les nouveaux comportements, usages et logiques induits par cette intégration interactive qu'opère le streaming de parties de jeu de rôle papier sur le spectateur.

Nous avons également pu observer qu'au-delà d'accomplir leur mission de divertissement, les émissions de parties de jeu de rôle sur table permettent d'accroître la notoriété et la popularité du jeu. Nous avons de plus constaté que ces émissions peuvent se révéler, dans certains cas, être des portes d'entrée dans le jeu. En effet, parmi les sujets qui ont répondu avoir découvert le jeu de rôle sur table via ces émissions, une majorité d'entre eux $(87,2 \%, S=34)$ déclarent avoir joué à ce jeu après cette découverte, poussés par ces mêmes émissions. Cette conclusion est toutefois à nuancer puisque 8,9\% $(\mathrm{S}=39)$ des sujets ont répondu avoir découvert le jeu de rôle papier grâce à ces émissions, ce qui demeure relativement faible. De plus, notre étude ne nous permet pas de déterminer la part d'individus qui ont connu le jeu par ce biais mais qui ne fréquentent pas les espaces socionumériques que nous avons retenu comme terrain de recrutement pour le questionnaire de l'enquête.

31 À l'aune de ces résultats, sans doute faut-il comprendre que l'entrée dans la pratique du jeu de rôle de table, malgré son déploiement dans les médias socionumériques, se déroule majoritairement par initiation directe sans la médiation d'émission ou de mise en scène de la pratique diffusée sur Internet. Autrement dit, l'arrivée de primo-joueurs se ferait 
encore aujourd'hui principalement par le biais d'une découverte et éventuellement d'un accompagnement de la part de joueurs de jeu de rôle de table dans leur entourage.

Jusqu'à présent nous nous sommes tout particulièrement intéressés au spectateur d'émissions de jeu de rôle papier mais qu'en est-il du créateur de ce type de contenus ? La perspective de se positionner, lors de futures recherches, du point de vue de ce dernier acteur pourrait être intéressante. En effet, l'occupation des nouveaux espaces et dispositifs socionumériques par le jeu de rôle sur table témoigne-t-elle initialement d'un désir de reconnaissance, de popularisation et d'attitude prosélyte ou bien n'est-elle destinée qu'aux spectateurs déjà joueurs, ce qui ferait de sa popularité croissante un épiphénomène involontaire de sa médiatisation?

\section{BIBLIOGRAPHIE}

BEAU, F. (dir.). (2007), Culture d'Univers. Jeux en réseau, mondes virtuels, le nouvel âge de la société numérique, Limoges, FYP Editions.

BEAUDOUIN, V. (2002), « De la publication à la conversation : Lecture et écriture électroniques », Réseaux, $\mathrm{n}^{\circ} 116$, pp. 199-225.

BESOMBES, N. (2015), « Du streaming au mainstreaming : mécanismes de médiatisation du sport électronique », in A. Obœuf (ed.), Sport \& Médias, Paris, CNRS Éditions, pp. 179-189.

BURGESS, J. et GREEN, J. (2009), YouTube. Digital media and society series, Cambridge, Polity Press.

CAÏRA, O. (2007), Jeux de rôle. Les forges de la fiction, Paris, CNRS Éditions.

CESAR, P. et GEERTS, D. (2011), Past, present, and future of social TV: A categorization , Conference at Consumer Communications and Networking Conference (CCNC), Las Vegas, IEEE, pp. 347-351, https://homepages.cwi.nl/ garcia/material/ccnc2011.pdf

DUMAZEDIER, J. (1974), Sociologie empirique du loisir, Paris, Éditions du Seuil.

DUMOUCHEL, R. (1991), « Le Spectacteur et le contactile ». Cinémas, 1(3), pp. 38-60.

FINE, G. A. (2002 [1981]), Shared Fantasy. Role-Playing Games as Social World, Chicago, Londres, University of Chicago Press.

GRANJON, F. (2012), Reconnaissance et usages d'Internet. Une sociologie critique des pratiques de l'informatique connectée, Paris, Presses des Mines.

REBILLARD, F. (2007), Le Web 2.0 en perspective, une analyse socio-économique de l'internet, Paris, L'Harmattan.

ROUX, U. (2016), « Jeu de rôle de table 2.0 : évolution d'une pratique ludique à l'ère du numérique ", Sciences du Jeu, $n^{\circ} 6$, https://sdj.revues.org/741.

SCHMITT, L. (2015), « Le « transmédia », un « label » promotionnel des industries culturelles toujours en cours d'expérimentation », Les Enjeux de l'information et de la communication, 2 (16), pp. 5-17. 
SHULGA, T. (2003), « Présence médiatisée et construction de l'espace d'interaction : Comparaison entre jeux de rôles classiques et MMORPG », Les Cahiers du numérique, 4 (2), pp. 101-115.

SJÖBLOM, M., et HAMARI, J. (2016), « Why do people watch others play video games? An empirical study on the motivations of Twitch users ", Computers in human behaviors, document non-publié.

TRÉMEL, L. (2001), Jeux de rôles, jeux vidéo, multimédia. Les faiseurs de mondes, Paris, Presses Universitaires de France.

VOELCKEL, A.-C. (1995), « Jouer ensemble. Approche biographique d'un loisir : le jeu de rôle », Sociétés Contemporaines, 1 (21), pp. 57-74.

WEISSBERG, J.-L. (1999), Présences à distance. Déplacement virtuel et réseaux numériques : pourquoi nous ne croyons plus à la télévision, Paris, L'Harmattan.

\section{NOTES}

1. Il faut bien distinguer le streaming en tant que solution technique du streaming en tant qu'activité culturelle (Sjöblom et Hamari, 2016). C'est à cette dernière activité que fait référence le présent travail.

2. Nous excluons donc dans cet article les autres types d'émissions sur le jeu de rôle sur table pour nous focaliser sur les retransmissions de parties.

3. "Le semi-loisir est une activité mixte où le loisir est mêlé à une obligation institutionnelle [...].» (Dumazedier, 1974, p. 97).

4. Un financement participatif (crowdfunding en anglais) visant la création du jeu de rôle papier tiré de l'émission éponyme a d'ailleurs vu le jour à la suite de son succès (source: https:// fr.ulule.com/aventures-le-jeu/).

5. Schmitt (2015) développe cette notion qui renvoie à une pluralité de pratiques d'écriture.

6. Le live streaming présente souvent un différé de quelques dizaines de secondes entre l'action du streameur et la réception de celle-ci par l'audience.

\section{RÉSUMÉS}

Il est observable depuis quelques années des diffusions en streaming de parties de jeu de rôle sur table. Le streaming, tel que nous l'entendons en tant qu'activité culturelle, se distingue des autres formes de diffusion de contenus vidéo en ligne par son haut niveau d'interaction qui en fait un canal communicationnel plus complet. Ce dispositif permet notamment aux spectateurs d'interagir entre eux mais aussi parfois avec les créateurs de contenus, et ce, souvent en temps réel. Ce faisant, le streaming étend à une audience les cadres de participation ainsi que les plans de présence du jeu de rôle papier. En intégrant le spectateur dans l'action ludique de manière toujours plus interactive, le streaming redéfinit l'espace de jeu dont les frontières peuvent parfois devenir ténues et poreuses avec le hors-jeu. Finalement, les logiques du jeu de rôle sur table se voient fréquemment repensées pour répondre aux nouveaux enjeux médiatiques. 
One can observe since a few years diffusions in streaming of tabletop role-playing games. Streaming, as a cultural activity, stands out from the other types of online video diffusions by its high level of interaction which makes it a more complete channel of communication. Streaming particularly allows viewers to interact between them and with the content creators also, and this, frequently in real time. In doing so, streaming extends to an audience the frameworks of participation and the planes of presence of pen-and-paper role-playing games. By integrating the viewer in the play in a more and more interactive way, streaming redefines the area of the game whose boundaries can sometimes become thin and porous with the "offgame". Finally, some tabletop role-playing game logics are frequently rethought to match with the new mediatic issues.

\section{INDEX}

Keywords : tabletop role-playing game, pen-and-paper role-playing game, digital era, social media, social television, streaming, spectator, spect-actor

Mots-clés : jeu de rôle sur table, jeu de rôle papier, ère numérique, média social, télévision sociale, streaming, spectateur, spectacteur 